\title{
EL PROBLEMA DE LA ERGODICIDAD EN LA MECÁNICA ESTADÍSTICA
}

\section{OLIMPIA LOMBARDI} CONICET

Universidad Nacional de Quilmes olimpiafilo@arnet.com.ar

RESUMEN: El propósito del presente artículo es evaluar en qué sentido y bajo qué condiciones la ergodicidad es relevante para explicar el éxito de la mecánica estadística. Se objeta la posición de quienes sostienen que la ergodicidad es irrelevante para tal explicación, y se señala que las propiedades ergódicas desempeñan diferentes papeles en la mecánica estadística del equilibrio y en la descripción de la evolución hacia el equilibrio: es posible prescindir de la ergodicidad en el primer caso pero no en el segundo. Sobre esta base, se reformularán las definiciones de ergodicidad y mezcla, relativizándolas a la macrovariable particular cuya evolución irreversible se desea describir. Finalmente, se enfatiza la importancia de tomar en cuenta la elaboración de modelos para evaluar la utilización de los métodos de Gibbs.

PALABRAS CLAVE: irreversibilidad, Boltzmann, Gibbs, modelos

SUMMARY: The aim of this paper is to consider in what sense and under what conditions ergodicity is relevant for explaining the success of Statistical Mechanics. We argue against those who claim that ergodicity is irrelevant to this explanation, by noting that ergodic properties play different roles in equilibrium Statistical Mechanics and in the description of the approach to equilibrium: it is possible to do without it in the first case but not in the second one. On this basis, we reformulate the definitions of ergodicity and mixing, relativizing them to the particular macrovariable whose irreversible evolution is to be described. Finally, we stress the relevance of taking into account model-construction for evaluating the use of Gibbs' methods.

KEY WORDS: irreversibility, Boltzmann, Gibbs, models

\section{Introducción}

Aún hoy, la mecánica estadística continúa generando profundos debates respecto de sus fundamentos teóricos. En este ámbito, uno de los problemas centrales consiste en justificar la introducción de probabilidades en un contexto clásico determinista. Precisamente para dar respuesta a este problema, Ludwig Boltzmann introdujo en 1871 la llamada "hipótesis ergódica", según 
la cual un sistema aislado recorre, en su evolución, todos los estados compatibles con su energía: en un sistema ergódico, cualquier punto representativo de su estado en el espacio de las fases pasa, a lo largo del tiempo, por todos los puntos de la hipersuperficie de energía constante. La idea es que, dado que en un sistema ergódico el punto representativo no queda "atrapado" en ninguna subregión de la hipersuperficie de energía constante sino que recorre toda su extensión, todos los puntos de dicha hipersuperficie son igualmente probables. De este modo, Boltzmann intentaba brindar una justificación dinámica de la equiprobabilidad sobre la región del espacio de las fases accesible al sistema.

Hoy se sabe que, por consideraciones dimensionales, la hipótesis ergódica en su formulación original no puede ser verdadera: dado que cualquier trayectoria en el espacio de las fases es unidimensional, no puede "cubrir" la hipersuperficie de energía constante cuya dimensión es superior a uno. No obstante, la idea implícita en el supuesto de Boltzmann puede conservarse mediante la hipótesis cuasiergódica que reemplaza la exigencia de que el punto representativo pase por todos los puntos de la hipersuperficie de energía constante por la exigencia de que pase por toda subregión de volumen finito incluida en la hipersuperficie de energía constante, y aclarando que la condición se cumple para los puntos pertenecientes a casi toda subregión de tal hipersuperficie excepto las subregiones de medida nula. De este modo se cumple que el punto representativo no permanezca atrapado en ninguna subregión de la hipersuperficie de energía constante, evitando la condición demasiado exigente de que pase por todos sus puntos a lo largo del tiempo.

En 1901, Josiah Willard Gibbs presentó un enfoque elegante y sistemático de la mecánica estadística que permitía derivar las relaciones entre variables termodinámicas a partir de las leyes dinámicas fundamentales sin introducir supuestos acerca de los detalles de las interacciones intermoleculares. Por su sencillez y elegancia, la formulación de Gibbs se convirtió con el tiempo en la herramienta teórica estándar en la mecánica estadística, cuya fecundidad en el tratamiento de problemas típicos de este 
ámbito es difícilmente cuestionable; en particular, el cálculo de los valores de equilibrio de las variables macroscópicas termodinámicas como promedios sobre el ensemble representativo es una estrategia de la cual ningún físico prescinde. Además de su función teórica original, en el enfoque gibbsiano la ergodicidad adquiere un nuevo papel al intervenir en la explicación de la evolución de los sistemas hacia el equilibrio termodinámico.

En la actualidad, la teoría ergódica se ha convertido en una teoría matemática que estudia las propiedades de los sistemas dinámicos desde una perspectiva exclusivamente formal. Sus resultados han permitido volver a analizar, con herramientas teórico-formales más precisas, el papel que cumple la ergodicidad en los fundamentos de la mecánica estadística. A la luz de estos resultados, durante las últimas décadas diversos autores comenzaron a cuestionar la relevancia explicativa que se asignaba a la ergodicidad desde la perspectiva tradicional. Este artículo se inserta en este contexto de discusión; su principal objetivo consiste en esclarecer en qué sentido y bajo qué restricciones la ergodicidad cumple un papel explicativo del éxito de la mecánica estadística de Gibbs. Para ello será necesario comenzar recordando los aspectos teóricos sobre los cuales se fundamenta la formulación gibbsiana pues, en algunos casos, el olvido de ciertas cuestiones básicas conduce a problemas conceptuales aparentemente irresolubles.

\section{Mecánica y termodinámica}

La mecánica estadística surge cuando se intenta explicar la termodinámica macroscópica en términos mecánicos microscópicos, bajo el supuesto de que las regularidades macroscópicas son resultado de las regularidades que rigen los componentes microscópicos de un sistema. Por lo tanto, cuando en este contexto se habla del sistema termodinámico y del sistema mecánico, no se alude a dos entidades independientes; por el contrario, se trata de un único sistema bajo diferentes descripciones: como un sistema termodinámico $S^{T}$ o como un sistema mecánico $S^{M}$. A fin de simplificar la discusión, me concentraré en sistemas ais- 
lados, esto es, sistemas que no intercambian materia ni energía con el medio.

\subsection{Descripción mecánica}

Sea $S^{M}$ un sistema mecánico aislado que, por tanto, conserva su energía mecánica $E_{M}$ constante a través del tiempo. Si $S^{M}$ está compuesto por $N$ subsistemas idénticos, cada uno de los cuales posee $n$ grados de libertad (traslacional, rotacional, etc.), su estado queda determinado por el valor de $2 n N$ variables. Llamando $q_{i}$ a las coordenadas generalizadas y $p_{i}$ a los momentos cinéticos generalizados de cada subsistema, el estado mecánico instantáneo $m(t)$ del sistema $S^{M}$ (microestado mecánico) queda definido por el valor de las $2 n N$ variables de estado:

$m(t)=\left(q_{i}(t), p_{i}(t)\right)=\left(q_{1}(t), q_{2}(t), \ldots, q_{n N}(t), p_{1}(t), p_{2}(t), \ldots, p_{n N}(t)\right)$

El estado instantáneo de $S^{M}$ suele representarse en el espacio de las fases correspondiente: espacio euclídeo abstracto de tantas dimensiones como variables de estado posea el sistema. En este caso, se trata de un espacio de las fases $\Gamma$ de $2 n N$ dimensiones, donde cada punto $x(t)$ representa un estado mecánico posible del sistema.

En el caso particular en el cual $S^{M}$ está compuesto por $N$ partículas puntuales de masa $m$, el estado mecánico de cada partícula queda determinado por el valor de seis variables: tres por sus coordenadas posicionales $q_{i}$ y tres por las componentes de su momento cinético $p_{i}=m q_{i}{ }^{2}$. Por lo tanto, el estado mecánico instantáneo $m(t)$ de $S^{M}$ queda definido por el valor de las $6 N$ variables de estado:

$m(t)=\left(q_{i}(t), p_{i}(t)\right)=\left(q_{1}(t), q_{2}(t), \ldots, q_{3 N}(t), p_{1}(t), p_{2}(t), \ldots, p_{3 N}(t)\right)$

La evolución temporal de $S^{M}$ se encuentra regida por las ecuaciones de Hamilton:

$$
d q_{i} / d t=\partial H / \partial p_{i} \quad d p_{i} / d t=-\partial H / \partial q_{i}
$$

donde el hamiltoniano $H\left(q_{i}, p_{i}\right)$ representa la energía mecánica total $E_{M}$ del sistema $S^{M}$. Las soluciones $q_{i}(t)$ y $p_{i}(t)$ representan la evolución temporal del sistema, dadas las condiciones iniciales $q_{i 0}$ y $p_{i 0}$. Puede demostrarse que tales ecuaciones 
cumplen las condiciones necesarias para asegurar la existencia y unicidad de sus soluciones para cada condición inicial: ${ }^{1}$ dado $\left(q_{i 0}, p_{i 0}\right)$, existe una única solución $\left(q_{i}(t), p_{i}(t)\right)$ de dichas ecuaciones, solución que describe la evolución mecánica del sistema. En el espacio de las fases $\Gamma$, tal evolución queda representada por una trayectoria que pasa por el punto $x_{0}=\left(q_{i 0}, p_{i 0}\right)$ en $t=0$; las condiciones de existencia y unicidad también pueden expresarse en lenguaje geométrico: para cada punto representativo del estado inicial, la trayectoria que en él se inicia existe y es única; además, dado que no hay restricciones para fijar el estado inicial del sistema $S^{M}$, las trayectorias no pueden cortarse en ningún punto, es decir, no existe ningún estado mecánico a partir del cual el sistema evolucione temporalmente según dos o más trayectorias posibles.

Dado que $S^{M}$ es un sistema aislado, $H\left(q_{i}, p_{i}\right)=E_{M}$ es una constante de movimiento del sistema que define una hipersuperficie $\Gamma_{E} \subset \Gamma$ de dimensión $d-1$, donde $d=2 n N$ es la dimensión del espacio de las fases:

$$
\Gamma_{E}=\left\{x=(q, p): H(q, p)=E_{M}\right\}
$$

Por lo tanto, todas las posibles evoluciones del sistema estarán representadas por trayectorias incluidas en $\Gamma_{E}$. En algunas ocasiones resulta conveniente representar la evolución dinámica del sistema $S^{M}$ mediante un operador $U_{t}$ tal que:

$$
\left(q_{i}(t), p_{i}(t)\right)=U_{t}\left(q_{i 0}, p_{i 0}\right)
$$

En el lenguaje del espacio de las fases:

$$
x(t)=U_{t} x_{0}
$$

${ }^{1}$ Dada una ecuación diferencial ordinaria de primer orden, si se cumplen las siguientes condiciones: (i) puede expresarse en su forma normal $d x / d t=$ $F(x, t)$; (ii) $F(x, t)$ y $d F / d x$ son continuas en cierto dominio $D$ del plano $O x t$; y (iii) $\left(x_{0}, t_{0}\right)$ es un punto de tal dominio, entonces existe una solución única $x(t)$ de tal ecuación diferencial que satisface $x=x_{0}$ para $t=t_{0}(\mathrm{cfr}$. Piskunov 1994). 
Un importante resultado que se cumple en este tipo de sistemas mecánicos - conservativos - es el teorema de Liouville. Sea $\rho(q, p)$ una función que define una medida $\mu$ sobre $\Gamma$ tal que:

- $\mu(\emptyset)=0$

- $\mu(\Gamma)=1$

- Si $A \subset \Gamma$ у $B \subset \Gamma$ у $A \cap B=\emptyset$, entonces $\mu(A \cup B)=$ $\mu(A)+\mu(B)$

La medida de un conjunto $A \subset \Gamma$ se define:

$$
\mu(A)=\int_{A} \rho(q, p) d \Gamma \quad \text { donde } d \Gamma=d q_{i} d p_{i}
$$

El teorema de Liouville demuestra que:

$$
\partial \rho / \partial t=-\sum\left(\partial \rho / \partial q_{i} d q_{i} / d t+\partial \rho / \partial p_{i} d p_{i} / d t\right)=0
$$

Esto implica que la medida $\mu$ se preserva a través de la evolución:

$$
\mu\left(\mathrm{U}_{t} A\right)=\mu(A)
$$

Intuitivamente, si se parte de una densidad $\rho$ cuyo soporte se encuentra confinado en cierta región del espacio de las fases, a través de la evolución tal región inicial puede deformarse y tornarse tan "filamentosa" como para extenderse hasta zonas distantes en el espacio de las fases, pero su volumen permanece siempre constante.

\subsection{Descripción termodinámica}

Sea $S^{T}$ un sistema termodinámico aislado; por lo tanto, su energía termodinámica $U$ se mantiene constante con el tiempo. El estado termodinámico $M(t)$ de $S^{T}$ en el instante $t$ queda definido por un conjunto de variables termodinámicas, entre las cuales pueden distinguirse dos grupos: 
- Las variables $C_{i}$ consideradas independientes en tanto controlables experimentalmente: su valor es fijado externamente con independencia de la situación particular en la que se encuentra $S^{T}$. Tales variables, que funcionan como parámetros, se denominan vínculos [constraints]: un ejemplo típico es el volumen de un sistema confinado en un recipiente rígido.

- Las variables dependientes $F_{i}$, cuyo valor se ajusta al de las primeras según ciertas relaciones termodinámicas conocidas. Ejemplos de tales variables son la presión y la densidad de un sistema.

Se define como equilibrio termodinámico al estado $M_{e q}$ para el cual las variables termodinámicas $F_{i}$ mantienen su valor constante con el tiempo: $d F_{i e q} / d t=0$. Se define la entropía $S$ del sistema en el estado de equilibrio $M_{e q}$ como:

$$
d S=d U / T
$$

donde $T$ es la temperatura absoluta y $d U$ representa un incremento diferencial de energía que conduciría al sistema a través de sucesivos estados de equilibrio.

Empíricamente se observa que cualquier sistema termodinámico aislado $S^{T}$ inicialmente fuera del equilibrio, evoluciona irreversiblemente hacia el equilibrio: las variables termodinámicas $F_{i}(t)$ varían su valor con el transcurso del tiempo hasta alcanzar su valor de equilibrio $F_{\text {ieq }}$, y a partir de allí mantienen su valor constante; nunca se observa la evolución inversa. En termodinámica se cuenta con distintas ecuaciones dinámicas no $t$-invariantes ${ }^{2}$ (por ejemplo, la ley de Fourier de difusión del

${ }^{2}$ Una ley dinámica (ecuación) es $t$-invariante si es invariante ante la inversión del signo de la variable $t$, y de todas las variables dinámicas involucradas. El concepto de $t$-invariancia también puede caracterizarse en términos de las evoluciones dinámicamente posibles respecto de una ley dinámica, esto es, soluciones de la ecuación correspondiente. Una evolución — secuencia de estados - $e_{i} \rightarrow e_{j}$ es dinámicamente posible respecto de la ley $L$ si es consistente con $L$, es decir, si queda representada por una solución de $L$. Llamando $\mathbf{T}(e)$ al estado temporalmente invertido respecto de $e$, la ley $L$ es $t$-invariante 
calor o la ley de Fick de difusión de materia) que describen la variación temporal irreversible del valor de las variables termodinámicas $F_{i}(t)$.

El segundo principio de la termodinámica expresa de un modo general la evolución irreversible de los sistemas termodinámicos aislados. Si el sistema $S^{T}$ evoluciona desde un estado de equilibrio $M_{e q 1}$ con entropía $S_{1}$ hasta un estado de equilibrio $M_{e q 2}$ con entropía $S_{2}$, se cumple que:

$$
S_{2}>S_{1}
$$

donde $S_{1}$ y $S_{2}$ son funciones de estado del sistema, esto es, no dependen de la particular evolución temporal que describe el sistema al pasar de $M_{e q 1}$ a $M_{e q 2}$.

\subsection{Conexión de ambas descripciones}

Bajo el supuesto de que ambas descripciones refieren a un único sistema, se impone el intento de relacionar de algún modo las variables termodinámicas macroscópicas de $S^{T}$ con las variables mecánicas microscópicas de $S^{M}$, de modo tal que las evoluciones temporales de las $F_{i}$ así como las relaciones entre ellas puedan explicarse en términos de las $q_{i}$ y $p_{i}$ y sus evoluciones temporales.

Aquí es importante recordar que si bien contamos con dos descripciones de un mismo sistema, sólo tenemos acceso empírico directo a las variables macroscópicas, cuyo valor puede determinarse por medición sobre el sistema (o calcularse de modo directo a partir de variables directamente medibles). Por el contrario, las variables microscópicas no pueden ser medidas debido al carácter inobservable de los subsistemas componentes; a ello se agrega el elevado número de grados de libertad del sistema - bajo su descripción $S^{M}$ - en la mayor parte de los casos de aplicación. Por lo tanto, sólo nos resta construir un modelo mecánico del sistema: se postula cierta estructura del sistema,

cuando se cumple lo siguiente: la evolución $e_{i} \rightarrow e_{j}$ es dinámicamente posible respecto de $L$ sii la evolución temporalmente invertida $\mathbf{T}\left(e_{j}\right) \rightarrow \mathbf{T}\left(e_{i}\right)$ es también dinámicamente posible respecto de $L$ ( $c f r$. Savitt 1995). Sobre el concepto de irreversibilidad nos detendremos más adelante. 
así como las características de los subsistemas componentes. La relación que se establezca entre el plano macroscópico y el microscópico será, entonces, una relación entre la descripción macroscópica $S^{T}$ del sistema y la descripción microscópica $S^{M}$ de un modelo mecánico de dicho sistema (volveremos sobre este punto en la sección 8).

El primer paso para conectar ambas descripciones consiste en comprobar que ciertas variables termodinámicas pueden identificarse sin inconvenientes con variables definidas en el plano mecánico. Por ejemplo, el volumen del sistema termodinámico $S^{T}$ puede asimilarse al volumen dentro del cual queda confinado el movimiento mecánico de los subsistemas componentes del sistema mecánico $S^{M}$. Sin embargo, en otros casos la relación no es tan directa: es necesario postular una asociación físicamente significativa entre las variables termodinámicas y las variables mecánicas. Un ejemplo sencillo es el de la energía en un sistema aislado: dado que no existe intercambio de energía con el entorno, la energía termodinámica de $S^{T}$ puede asimilarse a la energía mecánica de $S^{M}$ - energía cinética de movimiento más energía potencial de interacción. Otro ejemplo es el de la presión: si se considera el sistema bajo un modelo mecánico de partículas que colisionan con las paredes del recipiente que las contiene, la presión termodinámica de $S^{T}$ puede asociarse con la velocidad de transferencia de momento cinético a las paredes del recipiente por unidad de área y unidad de tiempo, debida al choque de las partículas de $S^{M}$. En general, se supone que el valor de las variables termodinámicas $F_{i}$ queda unívocamente determinado por el microestado mecánico del sistema; por lo tanto, a cada $F_{i}$ de $S^{T}$ (con excepción de la temperatura absoluta y la entropía) se asocia una función del estado mecánico de $S^{M}$. En otras palabras, para cada $F_{i}$ se define sobre el espacio de las fases $\Gamma$ una función de fase [phase function] $f_{i}: \Gamma \rightarrow \Re$ tal que asigne a cada punto de $\Gamma$ el valor de $F_{i}$ correspondiente. Dada su definición, $f(x)$ es una función de muchos-a-uno, pues toma el mismo valor para muchos microestados diferentes, esto es, para muchos puntos del espacio de las fases. 
A fin de aclarar los conceptos, concentraremos la atención en un ejemplo físico particular. Sea un gas confinado en la mitad izquierda de un recipiente rectangular de paredes perfectamente aislantes. Empíricamente se observa que, al retirar el tabique divisor entre las dos mitades, el gas comienza a difundirse de modo tal que, luego de cierto intervalo - tiempo de relajación del sistema - acaba distribuyéndose en todo el volumen accesible y en esta situación permanece; nunca se observa la evolución inversa - el gas concentrándose espontáneamente en la mitad izquierda del recipiente.

Según la descripción termodinámica, nos encontramos ante un sistema $S^{T}$, inicialmente en el estado de equilibrio $M_{e q 1}$, confinado en un volumen $V_{T 1}$ y con energía termodinámica $U$; un tiempo después de retirado el tabique divisor, $S^{T}$ adopta un nuevo estado de equilibrio $M_{e q 2}$ con volumen $V_{T 2}$ y manteniendo el valor de $U$ constante. Una variable macroscópica que pone de manifiesto el comportamiento irreversible del sistema es la densidad $\delta$ del gas en la mitad izquierda del recipiente: en el estado de equilibrio inicial, tendrá un valor $\delta\left(M_{e q 1}\right)$; al retirar el tabique divisor, irá disminuyendo con el tiempo hasta alcanzar el valor $\delta\left(M_{e q 2}\right)$ inferior al inicial. Sobre la base del segundo principio puede afirmarse que:

$$
S\left(M_{e q 2}\right)>S\left(M_{e q 1}\right)
$$

Suponiendo que se trata de un gas altamente diluido, podría postularse el siguiente modelo mecánico del sistema: $S^{M}$ es un sistema aislado respecto de fuerzas exteriores, compuesto por $N$ partículas puntuales de igual masa que no colisionan entre sí, pero que lo hacen contra las paredes del recipiente de volumen $V_{M}$ que las contiene. Si se desprecia la interacción gravitatoria entre partículas y se supone que las colisiones contra las paredes del recipiente son perfectamente elásticas, cada partícula se mueve a cierta velocidad constante entre colisiones, las cuales modifican la dirección y el sentido de la velocidad pero no su módulo. El microestado mecánico del sistema en el instante $t$ queda representado por un punto $x(t)=\left(q_{i}(t), p_{i}(t)\right)$ en el espacio de las fases $\Gamma$ de $6 N$ dimensiones, y su evolución queda 
representada por una trayectoria en dicho espacio, confinada en la hipersuperficie $\Gamma_{E}$ correspondiente a la energía mecánica total del sistema, $E_{M}$.

En la situación particular planteada, al retirarse el tabique divisor entre las dos mitades del recipiente, el movimiento mecánico de las partículas, inicialmente restringido a un volumen $V_{M 1}$, pasa a encontrarse restringido a un volumen $V_{M 2}>V_{M 1}$. Aquí, el volumen $V_{T}$ del gas puede identificarse con el volumen $V_{M}$ en el que se encuentra confinado el movimiento mecánico de las partículas $\left(V_{T}=V_{M}=V\right)$. Dado que el sistema se encuentra perfectamente aislado, la energía termodinámica $U$ puede asociarse con la energía mecánica total $E_{M}\left(U=E_{M}=E\right)$. A su vez, bajo el modelo mecánico dado, la densidad $\delta$ del gas en la mitad izquierda del recipiente en cada instante puede asociarse a la masa total por unidad de volumen en dicha mitad, $n(t) m /(V / 2)$, donde $n(t)$ es el número de partículas que se encuentran en la mitad izquierda del recipiente en el instante $t$. Dado que $n(t)$ queda unívocamente determinado por el microestado del sistema, puede definirse una función de fase $f_{\delta}(x)$ que adjudica a cada punto del espacio de las fases el valor correspondiente de $\delta$.

\section{El enfoque de Gibbs}

La estrategia de Gibbs se basa en describir el comportamiento del ensemble representativo del sistema bajo estudio. Un ensemble es un conjunto de sistemas que poseen la misma microestructura que el modelo mecánico del sistema bajo estudio, que están sometidos a los mismos vínculos externos, pero que se encuentran distribuidos sobre los diferentes microestados posibles. El microestado instantáneo de cada sistema del ensemble se representa mediante un punto en el espacio de las fases; la situación del ensemble como un todo queda representada por una "nube" de tales puntos representativos, uno por cada sistema del ensemble. Si el número $N$ de sistemas del ensemble es suficientemente alto, la situación del ensemble en cada instante puede especificarse mediante la densidad $\rho(t)$ de distribución de los puntos representativos en el espacio de las fases: 


$$
\rho(t)=\rho\left(q_{i}, p_{i}, t\right)
$$

cumpliéndose que:

$$
N=\int \ldots \int \rho\left(q_{i}, p_{i}, t\right) d q_{i} d p_{i}
$$

En general, $\rho$ se considera normalizada a la unidad:

$$
1=\int \ldots \int \rho\left(q_{i}, p_{i}, t\right) d q_{i} d p_{i}
$$

En este caso, $\rho$ define una medida que brinda la probabilidad por unidad de volumen de que un punto representativo de un sistema cualquiera del ensemble se encuentre en las diferentes regiones del espacio de las fases.

La función $\rho$ hace posible calcular los promedios, sobre todos los sistemas del ensemble, de cualquier magnitud que dependa de los microestados de tales sistemas. Por ejemplo, si consideramos una magnitud mecánica que define una función de fase $f: \Gamma \rightarrow \Re$, su promedio en fase en un instante dado se calcula:

$$
<f\left(q_{i}, p_{i}\right)>_{p}=\int \ldots \int f\left(q_{i}, p_{i}\right) \rho\left(q_{i}, p_{i}\right) d q_{i} d p_{i}=\int f(x) \rho(x) d x
$$

Desde este enfoque, se define equilibrio estadístico como la situación en la cual la probabilidad representada por $\rho$ y los promedios en fase son independientes del tiempo. Un modo sencillo de asegurar el equilibrio estadístico es construir un ensemble para el cual $\rho$ se distribuye uniformemente sobre todo el espacio de las fases:

$$
\rho\left(q_{i}, p_{i}\right)=c t e \rightarrow \partial \rho / \partial q_{i}=\partial \rho / \partial p_{i}=0 \rightarrow \partial \rho / \partial t=0
$$

En particular, para representar un sistema aislado — donde $E$ es una constante de movimiento - Gibbs recurre al ensemble microcanónico, que suele definirse de un modo totalmente general como:

$$
\begin{array}{ll}
\rho(x)=c t e & \text { para } x \in \Gamma_{E} \\
\rho(x)=0 & \text { para } x \notin \Gamma_{E}
\end{array}
$$

Sin embargo, en las presentaciones del tema no suele señalarse con suficiente claridad que, cuando nos referimos a un sistema 
aislado en particular, con los vínculos $C_{i}$ impuestos externamente, sus microestados posibles no son los representados por los puntos pertenecientes a $\Gamma_{E}$ : los sistemas del ensemble deben distribuirse uniformemente sobre los posibles microestados representados por puntos pertenecientes a $\Gamma_{E C} \subset \Gamma_{E}$, región accesible del espacio de las fases. Por lo tanto, en cada situación, dados los particulares vínculos impuestos al sistema bajo estudio, el ensemble microcanónico se define como:

$$
\begin{array}{ll}
\rho(x)=\text { cte } & \text { para } x \in \Gamma_{E C} \\
\rho(x)=0 & \text { para } x \notin \Gamma_{E C}
\end{array}
$$

En este caso, el promedio en fase de cualquier función de fase $f(x)$ se calcula como:

$$
<f(x)>_{p}=\int_{\Gamma E C} f(x) \rho(x) d \Gamma
$$

Con estos elementos, el valor de equilibrio de una variable macroscópica se calcula en términos del promedio en fase de su función de fase asociada: a cada variable macroscópica $F_{i}(t)$ del sistema termodinámico $S^{T}$ — con excepción de la entropía y la temperatura absoluta - le corresponde una función de fase $f_{i}(x)$ sobre el espacio de las fases de $S^{M}$ tal que, para el ensemble microcanónico representado por $\rho(x)$, se cumple:

$$
F_{i e q}=\left\langle f_{i}(x)\right\rangle_{p}=\int_{\Gamma E C} f_{i}(x) \rho(x) d \Gamma
$$

Por su parte, en estado de equilibrio estadístico, la entropía de Gibbs $S_{G}$ se calcula como:

$$
S_{G}=-k \int_{\Gamma} \rho(x) \log \rho(x) d \Gamma
$$

Hasta aquí nos hemos referido a la situación de equilibrio. Pero, ¿cómo dar cuenta, desde la perspectiva gibbsiana, de la macroevolución de un sistema aislado hacia el equilibrio? ${ }^{3} \mathrm{Su}-$ póngase un sistema sometido a ciertos vínculos $C_{0}$, inicialmente

${ }^{3}$ Es cierto que la mecánica estadística de Gibbs trata esencialmente de la situación de equilibrio. No obstante, ya en su Elementary Principles in Statistical Mechanics Gibbs (1902) considera el caso de un sistema aislado en su evolución hacia el equilibrio, y su idea original la continuaron posteriormente otros autores. Como veremos más adelante, en la actualidad continúa el debate 
en equilibrio termodinámico; en esta situación, el sistema es adecuadamente representado por un ensemble microcanónico definido por:

$$
\begin{array}{ll}
\rho(x)=\text { cte } & \text { para } x \in \Gamma_{E C O} \\
\rho(x)=0 & \text { para } x \notin \Gamma_{E C O}
\end{array}
$$

y la entropía de Gibbs será:

$$
S_{G}=-k \int_{\Gamma E C 0} \rho(x) \log \rho(x) d \Gamma
$$

Si en $t_{0}$ se modifican los vínculos aplicados al sistema (por ejemplo, en el caso del gas confinado en la mitad izquierda del recipiente, se elimina el tabique divisor), el macroestado originalmente de equilibrio se convierte en un macroestado de no equilibrio que evoluciona hacia un nuevo macroestado de equilibrio determinado por los nuevos vínculos $C_{1}$ impuestos al sistema. En la descripción de Gibbs, el ensemble microcanónico original representado por $\rho(x)$ se convierte, en $t_{0}$, en un ensemble de no equilibrio representado por $\rho_{0}(x, t)$ cuyo soporte se encuentra confinado en $\Gamma_{E C 0}$. Este ensemble de no equilibrio debería evolucionar hacia un nuevo ensemble microcanónico definido por:

$$
\begin{array}{ll}
\rho_{1}(x)=\text { cte } & \text { para } x \in \Gamma_{E C 1} \\
\rho_{1}(x)=0 & \text { para } x \notin \Gamma_{E C l}
\end{array}
$$

donde $\Gamma_{E C 1}$ es la nueva hipersuperficie accesible dados los nuevos vínculos impuestos, y siendo $\Gamma_{E C 1}>\Gamma_{E C 0}$. En otras palabras, luego de un tiempo $t$ superior al tiempo de relajación, debería darse la evolución $\rho_{0}\left(x, t_{0}\right) \rightarrow \rho_{0}(x, t)=\rho_{1}(x)$.

entre los defensores de un enfoque boltzmanniano y los continuadores del enfoque de Gibbs (para una comparación entre ambos enfoques, $c f r$. Lombardi 2000). Puesto que en este artículo no adopto una perspectiva histórica, formularé el problema en términos conceptuales tal como se presenta en nuestros días (para detalles históricos, cfr. Brush 1976). 


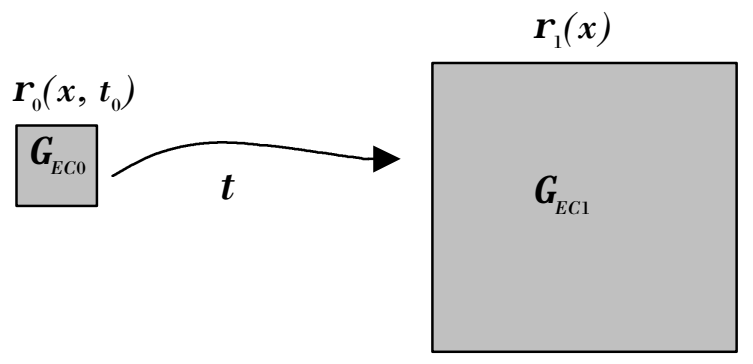

Sin embargo, tal situación no es posible. Los sistemas del ensemble inicial evolucionan según las leyes de la mecánica cumpliendo el teorema de Liouville de constancia de la medida en el espacio de las fases: la región $\Gamma_{E C 0}$ podrá deformarse y extenderse en la nueva región accesible del espacio de las fases pero, al mantener su volumen constante, no podrá cubrir de un modo efectivo la región $\Gamma_{E C 1}$ correspondiente al nuevo estado de equilibrio. En consecuencia, dada la validez del teorema de Liouville, la entropía $S_{G}$ se mantiene constante durante toda la evolución y, por lo tanto, no puede representar la entropía termodinámica $S$ regida por el segundo principio.

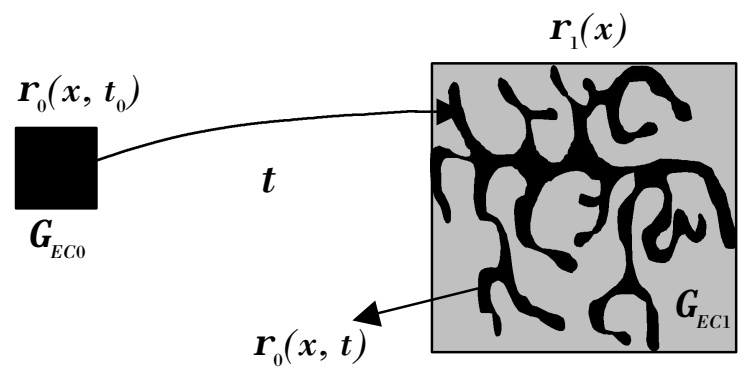

En el enfoque de Gibbs, lo que en realidad sucede es que la región inicial se ha distribuido y ramificado hasta el punto de cubrir de un modo aparentemente uniforme la región $\Gamma_{E C 1}$ correspondiente al nuevo macroestado de equilibrio. A fin de 
dar cuenta de la creciente deformación de la región $\Gamma_{E C 0}$ inicial, puede definirse una entropía de grano grueso [coarse grain] $S_{c g}$ : divídase el espacio de las fases en celdas y asígnese una probabilidad $P_{i}$ a cada una de ellas — probabilidad de que el punto representativo del microestado del sistema se encuentre en la celda $i ; S_{c g}$ se define como:

$$
S_{c g}=-k \Sigma P_{i} \log P_{i}
$$

y puede esperarse que aumente a través de la evolución, a medida que la región inicial vaya ingresando en mayor cantidad de celdas. No obstante, si un observador "perfecto" describiera la evolución del sistema inicialmente fuera del equilibrio a través del comportamiento de su ensemble representativo, observaría la creciente distorsión y ramificación en el espacio de las fases de la región $\Gamma_{E C 0}$ correspondiente al macroestado inicial, pero podría comprobar la validez del teorema de Liouville: nunca se alcanza una distribución uniforme sobre la región $\Gamma_{E C l}$ asociada al nuevo macroestado de equilibrio, pues el volumen de la región inicial permanece invariante durante toda la evolución. En consecuencia, el aumento de la entropía termodinámica $S$ que enuncia el segundo principio sólo puede asociarse al aumento de entropía de grano grueso $S_{c g}$, y ello implica una interpretación gnoseológica de la irreversibilidad.

En las discusiones acerca de la relevancia de la ergodicidad en la fundamentación de la mecánica estadística suele pasarse por alto que, en el contexto gibbsiano, las propiedades ergódicas cumplen un doble papel. En primer lugar, la ergodicidad justifica la elección de un ensemble microcanónico para representar el estado de equilibrio termodinámico, donde la función de distribución $\rho(x)$ adopta un valor constante en toda la región del espacio de las fases accesible al sistema. En segundo lugar, la ergodicidad interviene en la explicación de la evolución hacia el equilibrio: para que se produzca el aumento de la entropía de grano grueso $S_{c g}$, es necesario que el sistema sea mezclador, esto es, que la región inicial se deforme a través de la evolución; pero, a su vez, esto exige que el sistema sea ergódico, puesto que la propiedad de mezcla involucra un grado de inestabilidad 
superior a la ergodicidad, es decir, mezcla implica ergodicidad pero no a la inversa ( $c f r$. Lebowitz y Penrose 1973).

\section{El problema de la ergodicidad}

Las discusiones acerca del papel que cumple la ergodicidad en el esquema explicativo de Gibbs suelen circunscribirse al ámbito de la mecánica estadística del equilibrio, en particular, a la justificación de la estrategia gibbsiana de asimilar el valor de equilibrio de una macrovariable con el promedio en fase de su función de fase asociada mediante el ensemble microcanónico. Tradicionalmente, tal justificación se basa en el siguiente argumento: Dado que las mediciones macroscópicas insumen cierto tiempo, la magnitud medida no es el valor instantáneo de una variable macroscópica sino su promedio temporal. El intervalo de medición puede ser considerado muy corto a escala macroscópica, pero es muy largo a escala microscópica; por lo tanto, el promedio temporal sobre un intervalo finito puede asimilarse al promedio temporal infinito. A su vez, el teorema de Birkhoff asegura que, si el sistema es ergódico, para casi todo microestado inicial $x_{0}$, el promedio temporal infinito y el promedio en fase tienen el mismo valor. La idea que subyace al teorema es que, si el sistema es ergódico y se encuentra en su macroestado de equilibrio, la microevolución recorrerá, para $t \rightarrow \infty$, casi todos los puntos pertenecientes a la región del espacio de las fases accesible al sistema; por lo tanto, el promedio temporal de $f(x)$ para $t \rightarrow \infty$ puede igualarse a su promedio en fase. En consecuencia, el valor medido de la variable macroscópica puede aproximarse al promedio en fase de su función de fase correspondiente.

Este argumento tradicional, que suele incluirse en los textos sobre mecánica estadística, ha sido objeto de dos críticas principales:

- La cláusula "para casi todo $x_{0}$ " en el teorema de Birkhoff excluye los conjuntos de medida nula. Si el sistema se encuentra en un microestado representado por un punto perteneciente a tales conjuntos, el promedio temporal no 
está definido y, por tanto, el teorema de Birkhoff pierde su aplicabilidad. Ya Tolman (1938, pp. 68-69) objeta el papel del teorema de Birkhoff desde un punto de vista físico, al imaginar un gas confinado en un recipiente rectangular, y compuesto por partículas que se mueven paralelamente entre sí y respecto de dos de las caras del recipiente: el punto representativo de su microestado sólo podría recorrer una parte limitada de la región del espacio de las fases accesible al sistema. En consecuencia, a lo sumo podría afirmarse que la ergodicidad explica el éxito de los métodos de Gibbs en un sentido probabilístico, en particular, con probabilidad igual a uno.

- El intervalo que requiere una medición macroscópica no sólo no es infinito, sino que no puede considerarse como tal. En efecto, observamos la evolución de los sistemas hacia el equilibrio y efectuamos mediciones de magnitudes macroscópicas fuera del equilibrio: esto sería imposible si las mediciones siempre requirieran intervalos muy superiores al tiempo de relajación del sistema.

Estas objeciones fueron ya claramente formuladas por Sklar (1973), para luego ser retomadas por otros autores ( $c f r$. Malament y Zabell 1980; Leeds 1989; Earman y Rédei 1996). Sklar (1973, p. 209) agrega una nueva crítica cuando señala que el argumento tradicional no exige que el sistema posea un alto número de grados de libertad: un sistema de dos esferas en una caja es ergódico y, por tanto, responde al teorema de Birkhoff; sin embargo, no es un sistema al que pueda aplicarse la mecánica estadística de Gibbs. Otros autores que defienden un enfoque boltzmanniano de la mecánica estadística también manifiestan su insatisfacción frente al esquema explicativo de Gibbs por motivos análogos; por ejemplo, Bricmont (1995) insiste en la imposibilidad de brindar un sentido físico a la distinción micromacro en sistemas con pocos grados de libertad.

Pero entonces, ¿por qué funcionan los métodos de Gibbs? Malament y Zabell (1980) admiten que la asimilación de intervalos finitos a un intervalo infinito resulta inaceptable; no obstante, 
adjudican a la ergodicidad un papel limitado pero importante en la explicación del éxito predictivo de los promedios en fase. Según estos autores, el punto que debe justificarse es que la función de distribución microcanónica realmente representa la densidad de probabilidad de encontrar el sistema bajo estudio en un microestado particular. En efecto, $\rho(x)$ define una medida $\mu(A)$ sobre los subconjuntos $A$ de la región $\Gamma_{E C}$ del espacio de las fases accesible al sistema, tal que:

- es absolutamente continua, es decir, $\mu(A)=0$ para todos los subconjuntos $A$ de volumen nulo sobre $\Gamma_{E C}$.

- es invariante bajo la evolución $U_{t}$, es decir, para todo subconjunto medible $A, \mu(A)=\mu\left(U_{t} A\right)$.

Malament y Zabell concentran su atención en un corolario del teorema de Birkhoff según el cual, si el sistema es ergódico, la medida $\mu$ correspondiente al ensemble microcanónico es la única medida absolutamente continua e invariante sobre la región del espacio de las fases accesible al sistema. Sobre esta base, Malament y Zabell (1980, p. 345) concluyen que la ergodicidad brinda un importante argumento para considerar que el ensemble microcanónico efectivamente representa la probabilidad de que el sistema se encuentre en sus diferentes microestados posibles. $^{4}$

Si bien altamente plausible, este argumento es criticado por Earman y Rédei (1996), quienes admiten la validez de la estrategia de Malament y Zabell pero niegan su aplicabilidad a los sistemas macroscópicos de interés. En particular, Earman y Rédei (1996, p. 69) señalan que muchos de los sistemas típicos estudiados en la mecánica estadística son no ergódicos; y aun cuando el sistema bajo estudio sea sólo "un poco" no ergódico

${ }^{4}$ Malament y Zabell también señalan la importancia de que el sistema posea un elevado número de grados de libertad para el éxito de los métodos de Gibbs. En efecto, sobre la base de un resultado de Khinchin (1949) se demuestra que, si $f(x)$ tiene pequeña dispersión respecto de la medida $\mu$, la probabilidad de que su promedio en fase $\langle f\rangle_{p}$ difiera de su promedio temporal $\langle f\rangle_{t}$ también será muy baja y, por tanto, será altamente probable que los métodos de Gibbs "funcionen". 
- esto es, en una ínfima región del espacio de las fases-, el resultado de Birkhoff acerca de la unicidad de $\mu$ falla por completo y no sólo "un poco" (p. 71). De aquí, Earman y Rédei concluyen que "el uso de promedios en fase calculados sobre la medida microcanónica funciona; por lo tanto, la explicación de por qué funciona debe invocar mecanismos y propiedades no ergódicos" (Earman y Rédei 1996, p. 70).

El problema de determinar el papel que cumple la ergodicidad en la explicación del éxito de los métodos gibbsianos también fue abordado por otros autores. Por ejemplo, luego de una extensa crítica a la relevancia física del teorema de Birkhoff, Tolman (1938, pp. 63-70) abandona el argumento ergódico para justificar la equiprobabilidad y prefiere adoptar, como postulado adicional de la mecánica estadística, el principio de indiferencia, esto es, la hipótesis de iguales probabilidades a priori para regiones de igual medida en el espacio de las fases; este método "tiene la justificación a posteriori de conducir a conclusiones que concuerdan con los hechos empíricos" (Tolman 1938, p. 65). Esta estrategia de justificación adopta un carácter explícitamente pragmático en la argumentación de Sklar (1973): la distribución de los estados iniciales en las muestras de gas existentes en el mundo es tal que el esquema gibbsiano funciona mediante la medida microcanónica; "ésta es una cuestión de hecho, no de ley. Estos 'hechos' explican el éxito del método de Gibbs. Dicho claramente, constituyen la única explicación legítima de tal éxito" (Sklar 1973, p. 210). Si bien Sklar no lo menciona, su estrategia parece seguir la línea metodológica adoptada originalmente por el propio Gibbs con su actitud pragmática respecto de la mecánica estadística: el valor de la teoría reside exclusivamente en su capacidad de concordar con los resultados de la termodinámica fenomenológica; sobre esta base, la introducción de probabilidades no requiere otra explicación más que la utilidad de la teoría resultante ( $c f r$. Guttmann 1999, pp. 21-25).

La estrategia pragmática para justificar el éxito de los métodos gibbsianos prescindiendo de conceptos ergódicos puede resultar convincente en el marco de la mecánica estadística del 
equilibrio. Sin embargo, cuando se pasa a considerar la evolución hacia el equilibrio desde el marco teórico de Gibbs, no es tan sencillo prescindir de la ergodicidad. En equilibrio, el problema consiste en justificar un método que ha demostrado ampliamente su capacidad predictiva; si se prescinde de la hipótesis ergódica, puede adoptarse otro tipo de justificación sin que ello atente contra el éxito de tal método. Pero en el contexto de la evolución hacia el equilibrio, éste no es el caso, pues la explicación gibbsiana de la irreversibilidad depende esencialmente del carácter mezclador del sistema: si el sistema no es ergódico - y, por tanto, no es mezclador - el esquema teórico de Gibbs predice que no se producirá el aumento de la entropía de grano grueso asociada con la entropía macroscópica; en otras palabras, no se producirá el aumento de entropía postulado por el segundo principio. Por lo tanto, prescindir de la ergodicidad aquí no conduce simplemente, como en el caso del equilibrio, a conservar el método gibbsiano pero con una nueva justificación; en el contexto de la evolución hacia el equilibrio, prescindir de la ergodicidad equivale a rechazar la explicación gibbsiana de la irreversibilidad, esto es, a considerar incorrecta la asociación entre la entropía macroscópica y la entropía de grano grueso $S_{c g}$.

Esto explica la razón por la cual quienes adoptan, en una u otra variante, la línea teórica de Gibbs siempre subrayan la necesidad de que la microestructura del sistema posea un alto grado de inestabilidad para que se manifieste el fenómeno de la irreversibilidad. Uno de los primeros autores en señalar el papel esencial que cumple la inestabilidad dinámica para la irreversibilidad fue el físico ruso Krylov, al afirmar que "el proceso de mezcla es indispensable para que exista la relajación de los sistemas físicos, esto es, para hacer posible la existencia de una ley probabilística de la distribución de los estados que sea independiente del estado inicial y esté determinada por la fórmula de la fluctuación" (Krylov 1979, p. 19) ${ }^{5}$ Pero la idea de mezcla, si bien carente por completo de definición formal, fue

${ }^{5}$ Los trabajos de Krylov fueron originalmente publicados en la década de 1940. Una interesante discusión acerca de las tesis de este físico ruso puede encontrarse en Batterman 1990. 
ya introducida por Gibbs en el capítulo XII de su Elementary Principles in Statistical Mechanics con su famosa analogía de la gota de tinta en un vaso de agua (Gibbs 1902, pp. 144-145). ${ }^{6}$ En su ya clásico texto, Tolman retoma explícitamente la misma idea:

esta disminución [de $k \Sigma P_{i} \log P_{i}$ ] es resultado de la incapacidad de los puntos representativos del ensemble, originalmente presentes en alguna particular región pequeña pero finita $\delta q_{1} \ldots \delta p_{f}$ con densidad uniforme $\rho$, de moverse juntos como un todo compacto sin cambiar la "forma" de la extensión que ocupan. [...] las diferentes densidades de grano fino $\rho$, dentro de una celda del espacio de las fases correspondiente a un rango $E$ a $E+\delta E$, finalmente se mezclan como para darnos una densidad de grano grueso $P$ aproximadamente uniforme dentro de ese rango. (Tolman 1938, pp. 177-178)

Es interesante notar cómo Gibbs y Tolman, careciendo de elementos teóricos para relacionar ergodicidad y mezcla, creen posible prescindir de la ergodicidad en la situación de equilibrio para el cálculo del valor de las macrovariables como promedios en fase pero, no obstante, consideran la propiedad de mezcla como un elemento indispensable para la evolución irreversible del sistema hacia el equilibrio. Los matemáticos necesitaron más de treinta años para traducir en términos formales la intuición física de Gibbs; cuando finalmente lo hicieron, sus resultados iluminaron muchos aspectos relevantes para la discusión acerca del problema de la ergodicidad.

Sin embargo, también respecto de los procesos irreversibles surge el disenso cuando se intenta establecer el papel que cumple la ergodicidad. Algunos autores rechazan la explicación de Gibbs de la evolución hacia el equilibrio y prefieren adoptar la línea boltzmanniana, según la cual un sistema evoluciona desde

${ }^{6}$ Es interesante notar que el tratamiento que hace Gibbs de la irreversibilidad en el capítulo XII parece contradecir los primeros capítulos de su obra, donde el autor asume una actitud crítica frente al poder explicativo de la ergodicidad y asume una actitud pragmática acerca de la justificación de sus propios métodos. 
sus macroestados menos probables hacia sus macroestados más probables. Por ejemplo, Lebowitz (1993) desacredita la entropía de Gibbs como magnitud física relevante en la medida en que permanece constante durante la evolución del sistema; según el autor, sólo la entropía de Boltzmann — definida como una medida del número de microestados compatibles con cierto macroestado de equilibrio - captura la distinción entre las escalas microscópica y macroscópica. Desde una perspectiva similar, Bricmont (1995) sostiene que todo intento de "forzar" el aumento de la entropía de Gibbs mediante particiones de grano grueso genera la incorrecta impresión de que la irreversibilidad depende de tales particiones y, por tanto, es un fenómeno subjetivo. Según este autor, la ergodicidad no es condición ni necesaria ni suficiente para la irreversibilidad. No es condición suficiente puesto que hay sistemas ergódicos de pocos grados de libertad para los cuales no tiene sentido hablar de comportamiento irreversible. Pero la afirmación más fuerte es que la ergodicidad tampoco es condición necesaria, ya que existen evoluciones que, sin ser ergódicas, manifiestan un carácter inequívocamente irreversible. Lebowitz se refiere a los procesos irreversibles en un sentido análogo: "las características esenciales de la evolución no dependen de propiedades dinámicas específicas, como la positividad de los exponentes de Lyapounov, la ergodicidad o la mezcla" (Lebowitz 1993, p. 32).

En resumen, si bien es posible prescindir de la ergodicidad para explicar el éxito de los métodos de Gibbs en el contexto de la mecánica estadística del equilibrio, cuando se intenta explicar la evolución hacia el equilibrio el problema queda planteado con toda claridad. Mientras desde la perspectiva gibbsiana es indispensable que el sistema sea mezclador para manifestar un comportamiento irreversible, algunos autores sostienen que la ergodicidad no es condición necesaria para la irreversibilidad e incluso afirman la existencia de sistemas dinámicos no ergódicos que describen evoluciones irreversibles hacia el equilibrio. 


\section{Conceptos básicos de la teoría ergódica}

Para tratar el problema de la ergodicidad en términos actuales, es necesario contar con los principales conceptos y resultados que suministra la teoría ergódica en su presentación formal.

Sea un sistema dinámico $S$ definido por:

- El espacio de las fases $\Gamma$, compacto y metrizable.

- El flujo $U_{t}: \Gamma \rightarrow \Gamma$, con $t \in \Re$, tal que para todo $A \subseteq \Gamma$, $U_{0} A=A, U_{t+s} A=U_{s}\left(U_{t} A\right)$ y $U_{t} A=U_{-t}^{-1} A$.

- La medida $\mu$ sobre $\Gamma$, normalizada e invariante bajo el flujo $U_{t}$, y tal que para todo $A \subseteq \Gamma, \mu\left(U_{t} A\right)=\mu(A)$ para todo $t$.

Def. 1: El sistema $S$ es ergódico sii para todo $A \subseteq \Gamma$ tal que $\mu(A) \neq \emptyset$ y para casi todo $x \in \Gamma$ (excepto para conjuntos de medida nula), se cumple $\left\{U_{t} x\right\} \cap A \neq \emptyset$ para algún $t$.

Def. 2: El sistema $S$ es descomponible sii $\Gamma$ puede particionarse en dos o más regiones invariantes de medida no nula; por ejemplo, existen $A, B \subset \Gamma$ tales que $A \cap B=\emptyset, A \cup B=\Gamma, \mu(A) \neq \emptyset$, $\mu(B) \neq \emptyset$ y, para todo $t, U_{t} A \subseteq A$ y $U_{t} B \subseteq B$.

Teorema 1: El sistema $S$ es ergódico sii es no descomponible.

Def. 3: Sea el sistema $S$ y sea $f: \Gamma \rightarrow \Re$ una función de fase integrable:

- el promedio en fase de $f$ se define: $\langle f\rangle_{p}=$ $\int_{\Gamma} f(x) \mu(x) d \Gamma$

- el promedio temporal de $f$ se define: $\langle f\rangle_{t}=$ $\lim _{T \rightarrow \infty} 1 / T \int_{t 0}^{t 0+T} f\left(U_{t} x\right) d t$

Teorema 2 (teorema de Birkhoff): Sea el sistema $S$ y $f(x)$ una función de fase integrable sobre $\Gamma$ :

- Para casi todo $x \in \Gamma$ (excepto para conjuntos de medida nula), el promedio temporal de $f,\langle f\rangle_{t}$, existe y es independiente del instante inicial $t_{0}$. 
- Si el sistema $S$ es ergódico, para casi todo $x \in \Gamma$ (excepto para conjuntos de medida nula) se cumple que $<f>_{p}=<f>_{t}$.

Def. 4: El sistema $S$ es mezclador sii, para todo $A, B \subseteq \Gamma$, se cumple que $\lim _{|t| \rightarrow \infty} \mu\left(U_{t} A \cap B\right)=\mu(A) \mu(B)$.

Teorema 3: Sea el sistema $S, f(x)$ una función de fase integrable sobre $\Gamma$ y $\rho$ una medida absolutamente continua respecto de $\mu$ (esto es, para todo $A \subseteq \Gamma, \mu(A)=0 \Rightarrow \rho(A)=0$ ). Se define $\rho_{t}(A)=\rho\left(U_{t} A\right)$. Si el sistema es mezclador, $\rho_{t}$ converge a $\mu$ $\left(\rho_{t} \rightarrow \mu\right)$ para $|t| \rightarrow \infty$ en el sentido de que se cumple:

$$
\lim _{|t| \rightarrow \infty} \int_{\Gamma} f(x) \rho_{t}(x) d \Gamma=\int_{\Gamma} f(x) \mu(x) d \Gamma
$$

\section{6. ¿Sistemas no ergódicos?}

¿En qué tipo de sistema piensan los autores que, como Earman y Rédei, afirman que los sistemas típicos estudiados en mecánica estadística son no ergódicos? Tal vez un ejemplo de este tipo de sistema sea el caso de un gas cuyo modelo mecánico consiste en un conjunto de partículas puntuales que no colisionan entre sí, pero que lo hacen elásticamente con las paredes del recipiente que las contiene. ${ }^{7} \mathrm{Si}$ el gas se encuentra originalmente confinado en la mitad izquierda del recipiente, al retirarse el tabique divisor el valor de la densidad en dicha mitad disminuirá - salvo condiciones iniciales microscópicas excepcionaleshasta alcanzar un nuevo valor de equilibrio. No obstante, el sistema no es ergódico: dado que las partículas no colisionan entre sí, mantienen invariante el módulo de su velocidad; por lo tanto, no todas las regiones pertenecientes a la región $\Gamma_{E C}$ accesible al sistema son recorridas por el punto representativo.

${ }^{7}$ Un caso conocido de este tipo de modelo mecánico es el llamado "gas de Lorentz", compuesto de un gran número de partículas que no interactúan entre sí y que se mueven en un plano entre un arreglo periódico de dispersores [scatterers], ubicados de modo tal que una partícula no puede recorrer más que una distancia especificada entre colisiones. 
Sin embargo, en este caso la pregunta clave es: ¿esta forma de no ergodicidad es relevante respecto de la densidad? Para que la densidad en la mitad izquierda del recipiente disminuya hasta su valor de equilibrio sólo es relevante la posición de las partículas: la velocidad que posean las partículas en cada instante no influye en el valor de la densidad en dicho instante. Desde el punto de vista gibbsiano, la evolución irreversible de la densidad en la mitad izquierda del recipiente sólo exige que, a través del tiempo, el microestado del sistema adquiera - casi- todos sus posibles valores de posición. En el lenguaje del espacio de las fases, el punto representativo del microestado del sistema no debe permanecer confinado en ninguna subregión de medida no nula, ya no de la región accesible $\Gamma_{E C}$ en su totalidad, sino de una región $\Gamma_{p}$ incluida en $\Gamma_{E C}$ y de dimensión inferior a $\Gamma_{E C}$; tal región $\Gamma_{p}$ es resultado de la intersección entre $\Gamma_{E C}$ y el hipercilindro definido por el valor constante del módulo de la velocidad de las partículas. En definitiva, si bien el sistema no es ergódico respecto de toda la región accesible $\Gamma_{E C}$, sí es ergódico y, por tanto, no descomponible respecto de la subregión $\Gamma_{p} \subset \Gamma_{E C}$.

Si bien en el contexto restringido de la mecánica estadística del equilibrio, Earman y Rédei (1996, p. 74) se aproximan a esta idea cuando afirman que, a fin de preservar un papel explicativo para la ergodicidad, ya no es necesario referirse a ergodicidad y mezcla completas [full ergodicity, full mixing], sino a una ergodicidad "finita" y a una mezcla "finita", esto es, referidas a un conjunto finito de observables $\left\{\mathrm{O}_{\mathrm{i}}\right\}$. Los autores definen mezcla finita del siguiente modo:

Def. 5: El sistema definido por $\left(\Gamma_{E}, U_{t}, \mu\right)$ es mezclador respecto del conjunto finito de observables $\left\{\mathrm{O}_{\mathrm{i}}\right\}$ sii para toda medida $\rho$ absolutamente continua respecto de $\mu$ y tomando $\rho_{t}(A)=$ $\rho\left(U_{t} A\right)$, se cumple:

$$
\lim _{|t| \rightarrow \infty} \int_{\Gamma E} f_{\mathrm{Oi}}(x) \rho_{t}(x) d \Gamma_{E}=\int_{\Gamma} f_{\mathrm{Oi}}(x) \mu(x) d \Gamma_{E}
$$

y si bien no definen ergodicidad finita, es fácil suponer que lo harían del siguiente modo: 
Def. 6: El sistema definido por $\left(\Gamma_{E}, U_{t}, \mu\right)$ es ergódico respecto del conjunto finito de observables $\left\{O_{i}\right\}$ sii para casi todo $x \in \Gamma_{E}$ (excepto para conjuntos de medida nula) se cumple que:

$$
\left.<f_{\mathrm{Oi}_{\mathrm{i}}}>_{p}=<f_{\mathrm{Oi}_{\mathrm{i}}}\right\rangle_{t} \rightarrow \int_{\Gamma E} f_{\mathrm{Oi}}(x) \mu(x) d \Gamma=\lim _{T \rightarrow \infty} \mathrm{l} / T \int_{\mathrm{to}}^{\mathrm{to}+\mathrm{T}} f_{\mathrm{Oi}}\left(U_{t} x\right) d t
$$

En nuestro ejemplo, en lugar de ser completamente ergódico y mezclador, el sistema deberá ser ergódico y mezclador respecto del observable - macrovariable - 'densidad en la mitad izquierda del recipiente' para que el sistema explicativo gibbsiano funcione.

En primer lugar, cabe señalar que la posición que aquí adoptan Earman y Rédei no parece consistente con su afirmación anterior, según la cual la justificación del éxito de los métodos de Gibbs debe invocar "mecanismos y propiedades no ergódicos" (1996, p. 70): salvo que la ergodicidad finita y la mezcla finita sean consideradas "propiedades no ergódicas", no puede negarse que, aun desde esta perspectiva, ergodicidad y mezcla siguen cumpliendo un importante papel en la fundamentación de los métodos gibbsianos.

Pero un aspecto más interesante de la discusión es establecer el motivo por el cual Earman y Rédei consideran necesario introducir una diferencia conceptual entre ergodicidad y mezcla completas y ergodicidad y mezcla finitas. Los autores parecen considerar que la ergodicidad y la mezcla exigidas por el enfoque de Gibbs se refieren a toda la hipersuperficie $\Gamma_{E}$ del espacio de las fases; a fin de ajustar esta exigencia a las definiciones generales de ergodicidad y mezcla (definiciones 1 y 3 ), en lugar de definir el sistema dinámico mediante su espacio de las fases $\Gamma$ completo, lo hacen mediante la hipersuperficie $\Gamma_{E}$ (definiciones 5 y 6). Con ello, las definiciones de ergodicidad y mezcla completas (definiciones 1 y 3 ) como de ergodicidad y mezcla finitas (definiciones 5 y 6 ) se refieren al sistema dinámico $S=$ $\left(\Gamma_{E}, U_{t}, \mu\right)$, donde $\mu$ se define sobre $\Gamma_{E}$. Si bien esta estrategia no es objetable matemáticamente, desde un punto de vista físico parece bastante artificial: en el caso de nuestro sistema de partículas que no interactúan entre sí, al cambiar su energía total no nos encontraríamos ante el mismo sistema con diferente energía, sino ante un sistema diferente. La artificialidad de este 
enfoque se manifiesta claramente si se aplica al sencillo sistema de una única partícula libre en movimiento acelerado: en tanto su velocidad varía instantáneamente, su energía también se modifica, y la partícula cambiaría su identidad instante a instante.

Sin embargo, la estrategia matemática de Earman y Rédei se torna más difícilmente aceptable al recordar que, como fue señalado, cuando nos referimos a un sistema aislado en particular, con sus propios vínculos $C_{i}$ impuestos externamente, los microestados en los que puede encontrarse el sistema no son los representados por todos los puntos pertenecientes a $\Gamma_{E}$; por el contrario, los sistemas del ensemble microcanónico deben distribuirse uniformemente sobre los posibles microestados representados por puntos pertenecientes a $\Gamma_{E C} \subset \Gamma_{E}$, donde $\Gamma_{E C}$ es la región del espacio de las fases accesible al sistema dados su energía total y los vínculos que le son impuestos. En otras palabras, la región del espacio de las fases que resulta relevante no es toda la hipersuperficie $\Gamma_{E}$, sino la región accesible $\Gamma_{E C} \subset \Gamma_{E}$ : sólo sobre ella la mezcla y, por tanto, la ergodicidad se presentan como condición necesaria para el comportamiento irreversible del sistema. Pero $\Gamma_{E C}$ queda definida por los vínculos impuestos externamente. Por lo tanto, si adoptáramos la estrategia de Earman y Rédei de definir el sistema mediante la región del espacio de las fases relevante en cada caso, deberíamos aceptar que toda modificación en los vínculos impuestos modifica la identidad del sistema: en nuestro ejemplo, al eliminar el tabique divisor entre las dos mitades del recipiente, no diríamos que ha cambiado el volumen accesible al movimiento de los elementos del sistema, sino que nos encontramos ante otro sistema. Pero esta decisión metodológica se enfrenta al modo en que suele considerarse la identidad de los sistemas en la práctica científica: en nuestro ejemplo, al retirar el tabique divisor, el físico no considera que se encuentra ante un nuevo sistema, sino que el mismo sistema ha cambiado su volumen al modificarse uno de los vínculos impuestos desde el exterior y, con ello, se ha modificado la región del espacio de las fases accesible al sistema. Y aún más: si se aceptara que no se preserva la identidad del sistema con la modificación de los vínculos, sería necesario justificar por qué 
una ley física como el segundo principio establece una relación entre estados de equilibrio entre sistemas diferentes.

Sobre la base de estos argumentos, desde un punto de vista físico parece mucho más razonable definir un sistema dinámico mediante su espacio de las fases $\Gamma$ completo, cuyas características quedan determinadas por el número de grados de libertad del sistema y por las particulares coordenadas generalizadas de sus elementos componentes. A su vez, dado que la ergodicidad y la mezcla relevantes en esta discusión nunca se refieren al espacio de las fases $\Gamma$ completo, resulta más adecuado relativizar la definición misma de los conceptos de ergodicidad y mezcla respecto de cierta subregión $\Gamma_{\alpha}$ de $\Gamma$, del siguiente modo:

Def. 7: Dado un sistema $S$ definido por $\left(\Gamma, U_{t}\right)$, y dada una región $\Gamma_{\alpha} \subseteq \Gamma$ sobre la cual se define una medida $\mu, S$ es ergódico sobre $\Gamma_{\alpha}$ sii para todo $A \subseteq \Gamma_{\alpha}$ tal que $\mu(A) \neq \emptyset$ y para casi todo $x \in \Gamma_{\alpha}$ (excepto para conjuntos de medida nula), se cumple $\left\{U_{t} x\right\} \cap A \neq \emptyset$ para algún $t$.

Def. 8: Dado un sistema $S$ definido por $\left(\Gamma, U_{t}\right)$, y dada una región $\Gamma_{\alpha} \subseteq \Gamma$ sobre la cual se define una medida $\mu, S$ es mezclador sobre $\Gamma_{\alpha}$ sii para todo $A, B \subseteq \Gamma_{\alpha}$ se cumple que $\lim _{|t| \rightarrow \infty} \mu\left(U_{t} A \cap B\right)=\mu(A) \mu(B)$.

Esta relativización de los conceptos de ergodicidad y mezcla, no sólo preserva la identidad del sistema frente a modificaciones de los vínculos impuestos, sino que permite concentrar la atención en la subregión del espacio de las fases relevante en cada situación. Tal subregión puede quedar definida exclusivamente por los vínculos o, como en nuestro ejemplo, también por la particular relación entre la macrovariable de interés y las microvariables que definen el microestado del sistema.

Desde este nuevo punto de vista es posible comprender nuestro ejemplo de la densidad en la mitad izquierda de un recipiente desde una perspectiva diferente de la propuesta por Earman y Rédei. Para estos autores, tal densidad es el observable relevante $\mathrm{y}$, por tanto, es necesario definir el concepto de mezcla - y de ergodicidad - finita respecto de tal observable como un concepto diferente del de mezcla $-\mathrm{y}$ de ergodicidad - com- 
pleta. Desde nuestra perspectiva, por el contrario, no hay aquí dos conceptos diferentes de mezcla $-\mathrm{y}$ de ergodicidad-, sino un único concepto que se aplica a la región del espacio de las fases relevante en cada caso; en nuestro ejemplo se trata de la región $\Gamma_{p}$, incluida en la región accesible $\Gamma_{E C}$ y definida por el valor del módulo de la velocidad de las partículas que componen el sistema. En otras palabras, desde el enfoque gibbsiano, la irreversibilidad exige como condición necesaria que el sistema sea mezclador — ergódico- sobre $\Gamma_{p}$ según la definición 8 —definición 7-, donde $\Gamma_{\alpha}=\Gamma_{p}$.

En definitiva, en la formulación teórica de Gibbs las propiedades ergódicas del sistema — relativas a la región relevante del espacio de las fases - cumplen un importante papel en la explicación de la evolución irreversible hacia el equilibrio termodinámico en términos de la microdinámica subyacente. A su vez, dado que el sistema conserva sus propiedades ergódicas una vez alcanzado el equilibrio, la aplicabilidad del teorema de Birkhoff también adquiere su justificación. Por lo tanto, objetar la exigencia de ergodicidad para el éxito de los métodos de Gibbs señalando la existencia de sistemas típicos que no son siquiera ergódicos sobre $\Gamma_{E}$ resulta sumamente engañoso: ${ }^{8}$ es verdad que tales sistemas no son ergódicos ni mezcladores sobre toda la hipersuperficie $\Gamma_{E}$, pero sí lo son sobre una región más restringida del espacio de las fases, y ésta es la única condición necesaria de mezcla y ergodicidad que exige el enfoque de Gibbs.

\section{Volviendo al núcleo del problema}

Esta discusión acerca de las propiedades ergódicas que exige la perspectiva de Gibbs pone de manifiesto la importancia de no sucumbir a la seducción del formalismo matemático olvidando el problema físico que se intenta resolver. Earman y

${ }^{8}$ Esta crítica a los defensores del enfoque de Boltzmann se refiere exclusivamente al problema de la ergodicidad, pero no implica una completa adhesión al enfoque gibbsiano, el cual posee sus propias limitaciones como, por ejemplo, la necesidad de justificar la utilización de ensembles para explicar el comportamiento de sistemas singulares, o el carácter gnoseológico de la entropía de Gibbs. 
Rédei aceptan sin cuestionamiento la definición formal y totalmente general de mezcla que les suministra la teoría ergódica, y tratan de aplicarla a los sistemas para los cuales el enfoque de Gibbs ha mostrado su eficacia; al comprobar que tales sistemas no responden a la definición, inventan una nueva propiedad de mezcla definida a semejanza ya no de la definición general que brinda la teoría ergódica (definición 4), sino de una consecuencia de tal definición (teorema 3), consecuencia que, precisamente, asegura el éxito de la explicación gibbsiana. ¿Cómo se justifica la introducción de esta nueva propiedad? En la argumentación de los autores no parece haber otra justificación que el mero hecho de que la nueva propiedad asegura el éxito de los métodos de Gibbs. En otras palabras, si la pregunta es: “¿Por qué la mecánica estadística del equilibrio funciona?" (Earman y Rédei 1996, p. 74), una respuesta basada en una nueva propiedad cuya introducción se justifica exclusivamente en permitir que la mecánica estadística funcione resulta sospechosamente circular. Quienes consideramos que los problemas físicos exigen soluciones en términos físicos difícilmente podemos sentirnos satisfechos con tal respuesta.

Desde nuestra perspectiva, por el contrario, no es necesario definir una nueva propiedad de mezcla, sino sólo aplicar el concepto general de mezcla a una región particular del espacio de las fases. Aquí la pregunta relevante es: ¿cómo se justifica físicamente la elección de tal región? Sin duda, no apelando al éxito de la formulación gibbsiana. Para ello es necesario volver al núcleo del problema de la ergodicidad y recordar que el disenso adquiere el carácter de dificultad conceptual en el contexto de la evolución irreversible hacia el equilibrio. Por lo tanto, en primer lugar debe clarificarse el sentido preciso de los conceptos de reversibilidad e irreversibilidad que entran en juego en la discusión.

A diferencia del concepto de $t$-invariancia, aplicable a ecuaciones dinámicas, el concepto de reversibilidad no se aplica a ecuaciones o leyes sino a procesos. Un proceso $P$ compuesto por la sucesión temporal de eventos $a_{1}, a_{2}, \ldots, a_{n}$ es reversible si tal sucesión puede presentarse en ese orden o en el orden 
inverso; es irreversible si tal sucesión siempre se presenta en ese orden temporal, y nunca se observa en el sentido inverso $a_{n}, \ldots, a_{2}, a_{1}$. Cuando los conceptos de reversibilidad e irreversibilidad se expresan de este modo, $\operatorname{los}$ eventos $a_{i}$ son aspectos parciales de los estados de un sistema. Por ejemplo, en la mecánica, si los eventos considerados son las sucesivas posiciones de una partícula, la evolución temporal de la posición de la partícula es un proceso reversible; en la termodinámica, si los eventos considerados son las sucesivas densidades de un gas inicialmente confinado en la mitad izquierda de un recipiente a partir del momento en que se retira el tabique divisor entre las dos mitades, la evolución temporal de dicha densidad es un proceso irreversible. Es importante insistir en que los conceptos de reversibilidad e irreversibilidad no se refieren a los estados de un sistema, sino a la evolución temporal de alguna magnitud representada por una de las variables que define el estado del sistema. Esto es particularmente claro en la mecánica: si la sucesión de posiciones de una partícula en el tiempo es $x_{1}$, $x_{2}, \ldots, x_{n}$, tal sucesión puede presentarse en sentido temporal inverso, $x_{n}, \ldots, x_{2}, x_{1}$; en este caso, la ocurrencia de la sucesión inversa requiere la inversión temporal de los estados mecánicos de la partícula, de modo tal que la secuencia temporalmente invertida de estados no se compone de los estados originales sino de los estados temporalmente invertidos respecto de ellos: $T\left(e_{n}\right), \ldots, T\left(e_{2}\right), T\left(e_{1}\right)$. Por ello, el proceso del cual debería predicarse estrictamente la reversibilidad es la evolución temporal de la posición de la partícula, y no la evolución temporal de sus estados mecánicos.

En el caso termodinámico, el proceso al cual se aplica el predicado "irreversible" es la evolución temporal hacia el equilibrio de alguna propiedad observable representada por una variable macroscópica del sistema. ${ }^{9}$ En nuestro ejemplo, el predicado

${ }^{9} \mathrm{Si}$ bien tradicionalmente el problema de la irreversibilidad suele plantearse en términos del aumento de entropía, parece más natural hacerlo en términos de la evolución hacia el equilibrio de alguna propiedad observable del sistema, puesto que observamos cómo la densidad de un gas se vuelve uniforme o cómo se mezclan dos fluidos, pero no observamos en forma directa el flujo de entropía. 
irreversible se aplica al proceso generado por los sucesivos valores de la variable macroscópica 'densidad en la mitad izquierda del recipiente', valores que disminuyen progresivamente desde el instante en que se retira el tabique hasta alcanzar la situación de equilibrio. Pero cada variable dinámica macroscópica se asocia con una función de algunas de las variables microscópicas que definen el microestado del sistema, si bien no necesariamente con todas ellas; por ejemplo, si la macrovariable es la densidad, sólo son relevantes las posiciones de las partículas; pero si la macrovariable es la temperatura, las microvariables relevantes son las velocidades de las partículas. En consecuencia, en la explicación de la evolución irreversible de cierta variable macroscópica no es necesario que la propiedad de mezcla se refiera a todas las microvariables del sistema, incluidas aquellas que resultan irrelevantes respecto de la macrovariable considerada; por el contrario, sólo es necesario que el sistema sea mezclador respecto de las microvariables que intervienen en la función asociada a la macrovariable cuya evolución irreversible se pretende explicar.

El lenguaje del espacio de las fases permite expresar la misma condición en términos geométricos. Sea un sistema $S$ aislado con su descripción macroscópica $S^{T}$ y su descripción microscópica $S^{M}$. Sea $F(t)$ una variable macroscópica de $S^{T}$. Sean $x_{1}, \ldots, x_{d}$ las $d$ microvariables que definen el microestado de $S^{M}$ y mediante las cuales se construye el espacio de las fases $\Gamma$ de dimensión $d$. Supóngase que la macrovariable $F$ se asocia con una función de las primeras $k$ microvariables de estado: $f\left(x_{i}\right)$, con $1 \leq i \leq k$ y $k<d$. Para que el enfoque de Gibbs explique la evolución irreversible de $F(t)$ es condición necesaria que $S^{M}$ sea mezclador sobre una región $\Gamma_{k}$ incluida en la región $\Gamma_{E C}$ accesible al sistema, donde $\Gamma_{k}$ es resultado de la intersección entre $\Gamma_{E C}$ y la hipersuperficie definida por todas las combinaciones de los valores posibles de las restantes microvariables de estado $x_{j}$, con $k \leq j \leq d$. Un modo tal vez más sencillo de visualizar la misma condición consiste en descomponer el espacio de las fases $\Gamma$ en dos subespacios $\Gamma^{i}$ y $\Gamma^{j}$ construidos mediante las microvariables $x_{i}$ y $x_{j}$, respectivamente; para explicar la evolución 
irreversible de $F(t)$ es condición necesaria que la propiedad de mezcla se cumpla sobre la región $\Gamma_{E C}^{i}$ accesible al sistema, ahora definida sobre el subespacio $\Gamma^{i}$. En nuestro ejemplo, para que el esquema teórico de Gibbs explique la evolución irreversible de la densidad del gas en la mitad izquierda del recipiente es condición necesaria que la propiedad de mezcla se cumpla sobre la región $\Gamma_{E C}^{p}$ definida por la energía y los vínculos del sistema, pero donde $\Gamma_{E C}^{p}$ es una región del subespacio de posición $\Gamma^{p}$ obtenido de descomponer el espacio de las fases $\Gamma$ correspondiente al sistema.

Esta argumentación pone de manifiesto que la discusión acerca de la relevancia de las propiedades ergódicas en la teoría de Gibbs no puede perder de vista el problema físico que intenta resolver. La teoría ergódica, en cuanto formalismo matemático, brinda una excelente herramienta para precisar los conceptos involucrados en el debate, pero esto no implica que pueda utilizársela de un modo indiscriminado. Como toda teoría matemática, la teoría ergódica no puede brindar por sí misma la respuesta a un problema físico: su aplicación sólo será fructífera si previamente sus términos formales se interpretan con precisión a la luz de los aspectos fácticos del fenómeno físico bajo estudio. Cuando esta precaución metodológica se pierde entre la maraña de argumentos puramente formales, fácilmente pueden extraerse conclusiones inadecuadas $o$, al menos, irrelevantes respecto del problema físico en cuestión.

\section{El papel de los modelos}

Una pregunta totalmente diferente de las formuladas en las secciones anteriores es la que se refiere a las características de los sistemas físicos reales estudiados en la mecánica estadística en cuanto a su grado de inestabilidad: ¿̨cómo se aplican los resultados de la teoría ergódica en la práctica científica? Los críticos de la relevancia de la ergodicidad están en lo cierto cuando afirman que muchos sistemas estudiados en la mecánica estadística no son ergódicos y, por tanto, son descomponibles. En algunas situaciones, la descomponibilidad posee manifestaciones 
observacionalmente accesibles; éste es el caso, por ejemplo, de una fase sólida en equilibrio con una fase líquida o, en general, de cualquier fase en equilibrio con otra. En estas situaciones, los métodos de Gibbs se aplican no sobre toda la región del espacio de las fases accesible al sistema, sino sobre cada una de las subregiones en las cuales aquélla se descompone. No obstante, en muchos casos el sistema no manifiesta observacionalmente señal alguna de descomponibilidad; ¿cómo actúa el físico cuando se enfrenta a este tipo de sistemas? La estrategia usual es aplicar, de todos modos, los métodos de Gibbs bajo el supuesto de que el sistema es ergódico y posee las propiedades ergódicas más fuertes. Sin duda, esta estrategia no asegura el éxito predictivo. Si los métodos gibbsianos funcionan, ello brindará elementos en favor del supuesto de ergodicidad. Pero, ¿qué sucede cuando las predicciones no se confirman empíricamente? En este caso, la reacción del físico no será abandonar drásticamente el enfoque de Gibbs, sino buscar argumentos adicionales en favor de la descomponibilidad del espacio de las fases en subregiones donde los métodos de Gibbs sigan siendo aplicables. Como señala Quay:

un ejemplo clásico de este tipo de fracaso de la mecánica estadística se encuentra en los primeros tratamientos del $H_{2}$ puro. Como resultado de las marcadas diferencias entre la teoría y los resultados experimentales, se encontró que el espacio de las fases del $H_{2}$ debía dividirse en dos subespacios correspondientes al orto y al para hidrógeno. (Quay 1978, p. 54)

Estas consideraciones nos conducen a recordar un aspecto que frecuentemente se pasa por alto en las discusiones en torno a los fundamentos de la mecánica estadística. Cuando se intenta conectar la descripción mecánica $S^{M}$ y la descripción termodinámica $S^{T}$ de un mismo sistema, sólo se tiene acceso empírico directo a las variables macroscópicas correspondientes a $S^{T}$. Por el contrario, la descripción mecánica $S^{M}$ refiere a un modelo mecánico del sistema, construido sobre la base de postular cierta estructura microscópica, así como las caracterís- 
ticas de los subsistemas componentes. Por lo tanto, cuando nos enfrentamos a un sistema a cuya descripción termodinámica $S^{T}$ podemos acceder empíricamente y pretendemos aplicar los métodos de Gibbs, debemos necesariamente postular un modelo mecánico microscópico al cual referirá la descripción $S^{M}$. La construcción del modelo depende del conocimiento previo o de conjeturas acerca de la microestructura del sistema.

Esta etapa descriptiva permite aplicar los métodos de Gibbs, pero aún no asegura su éxito predictivo: bien puede suceder que no se obtengan resultados satisfactorios. Frente a esto, la situación más favorable sería aquella en la que pudiéramos demostrar formalmente que nuestro modelo es ergódico, e incluso que posee las propiedades ergódicas más fuertes; en este caso, la estrategia más razonable consistiría en intentar reformular el modelo, tal vez haciéndolo más complejo e incluyendo factores considerados irrelevantes en el modelo original. Pero, lamentablemente, en la práctica la situación dista de ser tan sencilla: la demostración formal de que un sistema posee ciertas propiedades ergódicas es una tarea de enorme complejidad matemática, y sólo se ha logrado en casos altamente idealizados a través de procedimientos sumamente complejos. Por lo tanto, prácticamente siempre trabajamos con modelos mecánicos cuyas propiedades ergódicas no podemos demostrar. Frente a ello habría dos actitudes posibles. $\mathrm{Si}$, atendiendo a las críticas, prescindiéramos por completo del supuesto de ergodicidad, deberíamos renunciar a utilizar una poderosa herramienta teórica como la gibbsiana para explicar las propiedades y, principalmente, el comportamiento dinámico del sistema macroscópico en su evolución hacia el equilibrio. Pero éste es un precio demasiado alto que los físicos suelen no estar dispuestos a pagar, al menos no desde el comienzo. Por ello, la estrategia más frecuente consiste en suponer que las propiedades ergódicas se cumplen y aplicar los métodos de Gibbs esperando obtener predicciones exitosas; si esto no sucede, se intenta reformular el modelo mecánico a la luz de argumentos físicos adicionales. De todos modos, esta estrategia tampoco asegura que se alcanzará una adecuación predictiva: si bien la mecánica estadística de Gibbs ha demostrado su capaci- 
dad para abordar múltiples y disímiles situaciones, hay muchos casos en los cuales aún no se ha podido aplicar exitosamente. ${ }^{10}$

Estos argumentos, referidos a la práctica científica en el contexto de la mecánica estadística, permiten afirmar que el supuesto de ergodicidad no constituye un ingrediente superfluo o estéril en la teoría gibbsiana. Por el contrario, el fracaso predictivo de los métodos de Gibbs bajo el supuesto de ergodicidad - no descomponibilidad - brindan información extremadamente útil acerca de la estructura microscópica del sistema, e incluso puede conducir a conjeturar ciertas propiedades inobservables de los subsistemas microscópicos componentes.

A su vez, los mismos argumentos permiten evaluar bajo una nueva luz el debate acerca del papel explicativo de la ergodicidad en el marco teórico de Gibbs. En las discusiones suele enfatizarse el éxito predictivo de los métodos gibbsianos pero olvidando sus limitaciones. Si bien tal éxito es innegable, no pueden ignorarse los casos en los que los resultados no son los previstos. Las críticas al papel explicativo de la ergodicidad basadas en la existencia de sistemas no ergódicos olvidan que, cuando el sistema es no descomponible, los métodos gibbsianos no son aplicables sobre toda la región del espacio de las fases accesible al sistema, sino que sólo lo son sobre cada una de las subregiones que resultan de la descomposición. Por consiguiente, la no ergodicidad de muchos sistemas de interés para la mecánica estadística no impugna el papel de las propiedades ergódicas en el marco explicativo de Gibbs. En definitiva, la exigencia de ergodicidad y de mezcla que impone el enfoque de Gibbs no asegura el carácter ergódico y mezclador de todos los sistemas relevantes en la mecánica estadística, sino sólo de aquellos donde se aplica exitosamente. Por lo tanto, mientras muchos autores formulan el problema preguntando "¿Por qué los métodos de Gibbs funcionan?", en realidad la pregunta adecuada es "¿Por qué los métodos de Gibbs funcionan cuando funcionan?", y en su respuesta las propiedades ergódicas cumplen un importante papel.

\footnotetext{
${ }^{10}$ Por ejemplo, los métodos gibbsianos todavía no se han podido aplicar exitosamente a fluidos de muy alta densidad.
} 


\section{Conclusiones}

Sin duda, los resultados formales de la teoría ergódica han permitido discutir los problemas asociados con la fundamentación de la mecánica estadística mediante argumentos mucho más precisos que los esgrimidos por los padres fundadores de la teoría. Sin embargo, en muchas discusiones tales resultados formales se aplican de un modo completamente general y no se relativizan a la luz del fenómeno físico que se pretende explicar. El poder de la herramienta matemática ha oscurecido la cuestión física.

La posición formulada en este artículo acerca del papel que cumplen las propiedades ergódicas en el esquema teórico de Gibbs cierra el camino a argumentos basados en la demostración "limpia" de teoremas generales. Nuestra perspectiva exige considerar, en cada sistema de interés, los argumentos físicos que conducen a escoger cierto modelo mecánico y los resultados predictivos que se obtienen de tal elección. Además, para cada modelo mecánico asociado al sistema bajo estudio, se impone el análisis del comportamiento de las microvariables relevantes respecto de la macrovariable cuya evolución se pretende describir. Es innegable que la incorporación de estos aspectos conceptuales y metodológicos hace más complejos los términos del debate. Pero si olvidamos el núcleo del problema físico de la ergodicidad, corremos el riesgo de extraviarnos en discusiones puramente formales acerca de propiedades abstractas y generales que, finalmente, los sistemas físicos suelen no poseer.

\section{BIBLIOGRAFÍA}

Batterman, R.W., 1990, "Irreversibility and Statistical Mechanics: A New Approach?", Philosophy of Science, vol. 57, pp. 395-419.

Bricmont, J., 1995, "Science of Chaos or Chaos in Science?", Physicalia Magazine, vol. 17, pp. 159-208.

Brush, S., 1976, The Kind of Motion We Call Heat, North Holland, Amsterdam.

Earman, J. y M. Rédei, 1996, "Why Ergodic Theory Does Not Explain the Success of Equilibrium Statistical Mechanics", British Journal for the Philosophy of Science, vol. 47, pp. 63-78. 
Gibbs, J.W., 1902, Elementary Principles in Statistical Mechanics, Yale University Press, New Haven.

Guttmann, Y.M., 1999, The Concept of Probability in Statistical Physics, Cambridge University Press, Cambridge.

Khinchin, A.I., 1949, Mathematical Foundations of Statistical Mechanics, Dover, Nueva York.

Krylov, N.S., 1979, Works on the Foundations of Statistical Physics, Princeton University Press, Princeton.

Lebowitz, J.L., 1993, “Boltzmann's Entropy and Time's Arrow", Physics Today, vol. 46, pp. 32-38.

Lebowitz, J.L. y O. Penrose, 1973, "Modern Ergodic Theory", Physics Today, vol. 26, pp. 23-29.

Leeds, S., 1989, "Malament and Zabell on Gibbs Phase Averages", Philosophy of Science, vol. 56, pp. 325-340.

Lombardi, O., 2000, "La interpretación de la irreversibilidad: Prigogine versus Gibbs", Diálogos, año XXXV, pp. 37-56.

Malament, D.B. y S.L. Zabell, 1980, "Why Gibbs Phase Averages Work - The Role of Ergodic Theory", Philosophy of Science, vol. 47, pp. 339-349.

Piskunov, N., 1994, Cálculo diferencial e integral, Limusa, México.

Quay, P.M., 1978, "A Philosophical Explanation of the Explanatory Functions of Ergodic Theory", Philosophy of Science, vol. 45, pp. 47-59.

Sklar, L., 1973, "Statistical Explanation and Ergodic Theory", Philosophy of Science, vol. 40, pp. 194-212.

Savitt, S.F., 1995, "Introduction", en S.F. Savitt (comp.), Time's Arrows Today, Cambridge University Press, Cambridge.

Tolman, R.C., 1938, The Principles of Statistical Mechanics, Clarendon Press, Oxford.

Recibido el 13 de septiembre de 2002; aprobado el 8 de enero de 2003. 